\title{
Konventionelle Röntgendiagnostik der Patella: Einstelltechnik, Tipps und Tricks
}

\section{Convential Diagnostic Radiology of the Patella: Adjustment Techniques, Tips, and Tricks}

\author{
C. Becker
}

Bei Arbeit, Sport und Spiel kann es passieren. Ein plötzlicher Schmerz im Knie, ein Sturz oder Ähnliches. Eine der möglichen Ursachen ist eine Verletzung, Beeinträchtigung oder Beteiligung der Patella. Um hier Klarheit zu schaffen, sind meist spezielle Röntgenaufnahmen notwendig. In

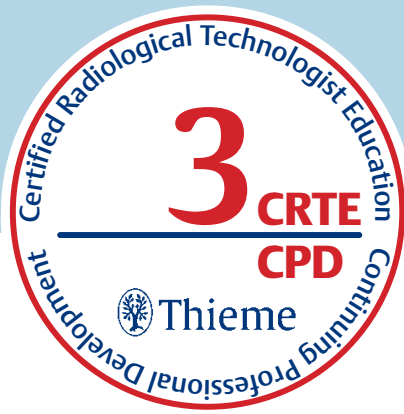
diesem Artikel werden Patella tangential, Patella Defilée und Patella nach Merchant vorgestellt. Es wird auf die richtige Einstellung, Fehler und deren Behebung eingegangen.

\section{Anatomie}

Das Knie ist ein Dreh-Scharnier-Gelenk. Die am Gelenk beteiligten Knochen sind Femur und Tibia ( Abb. 1 u. $\odot$ Abb.2). Angrenzend befinden sich noch die Articulatio tibiofibularis und die Gelenkverbindung zur Patella (Articulatio femoropatellaris). Es wird von Bändern, Muskeln und Sehnen gehalten und stabilisiert. Vornehmlich sind das die Kreuzbänder, die Seitenbänder, der M. quadriceps, die Quadrizepssehne und die Patellasehne.

Die Patella, das größte Sesambein des menschlichen Körpers, ist hierbei ein sehr wichtiger Gelenkteil ( Abb.3 u. Abb.4). Sie ist in die Sehne des M. quadriceps eingebettet und fungiert als „Umlenkrolle“, um bei der Kniebeugung den Zug des M. quadriceps umzulenken. Dadurch wird zum einen die Sehne geführt und der Widerstand verringert, wenn die Sehne über den Knochen gleitet, zum anderen erhöht sich die Kraftwirkung über den verlängerten Hebelarm. Nach distal ist sie über die Patellasehne mit der Tibia verbunden.

Die Form der Patella ist von vorne betrachtet scheibenförmig. Von axial betrachtet entsteht der Eindruck eines Keils ( $\bullet$ Abb.5). Am Femur zeigt sich ventral eine Rinne zwischen den Kondylen. In dieser Rinne, dem sog. Gleitlager oder Facies patellaris, gleitet die Patella bei der Beugung und Streckung über den Femur ( $\bullet$ Abb.6).
Nach proximal weist die Patella eine breitere Kante, die Basis patellae, und nach distal eine Spitze, die Apex patellae, auf. Die Rückseite, die Facies posterior, ist mit Knorpel überzogen und erleichtert dadurch die Gleitfähigkeit. An der Basis setzt der M. quadriceps mit der Quadrizepssehne an. Er besteht aus 4 Anteilen ( $\bullet$ Abb. 7):

- Rectus femoris (violett)

- Vastus intermedius (grau)

- Vastus lateralis (hellblau)

Vastus medialis (rot)

An der Apex setzt die Patellasehne an. Der Vastus medialis ist maßgeblich daran beteiligt, dass die Patella auf ihrer Bahn gehalten wird.

Bei der knöchernen Ausbildung der Patella können verschiedene Ausformungen auftreten. Eine eher flache Form kann zu Problemen führen und einen stabilen Lauf im Gleitlager beeinträchtigen. Sie werden eingeteilt nach Wiberg ( $\bullet$ Abb.8).

Neben solchen anlagebedingten Unterschieden gibt es weitere individuelle Unterschiede, die meist anlagebedingt oder Folge eines Traumas sind, z. B.:

degenerative Veränderungen

- Verletzungen oder Frakturen

- Patelladysplasie

- Patella partita ( $\bullet$ Abb.9)

Osteochondrosis dissecans

Die Patella ist das größte Sesambein des menschlichen Körpers. 
Abb. 1 Anatomie Knie lateral. Femur: hellrot, Femurkondylen: rosa, Patella: gelb, Fibula: hellblau, Fibulaköpfchen: dunkelblau.

Abb.2 Anatomie Knie a.-p. Femur: hellrot, Femurkondylen: rosa, Patella: gelb, Fibula: hellblau, Fibulaköpfchen: dunkelblau.
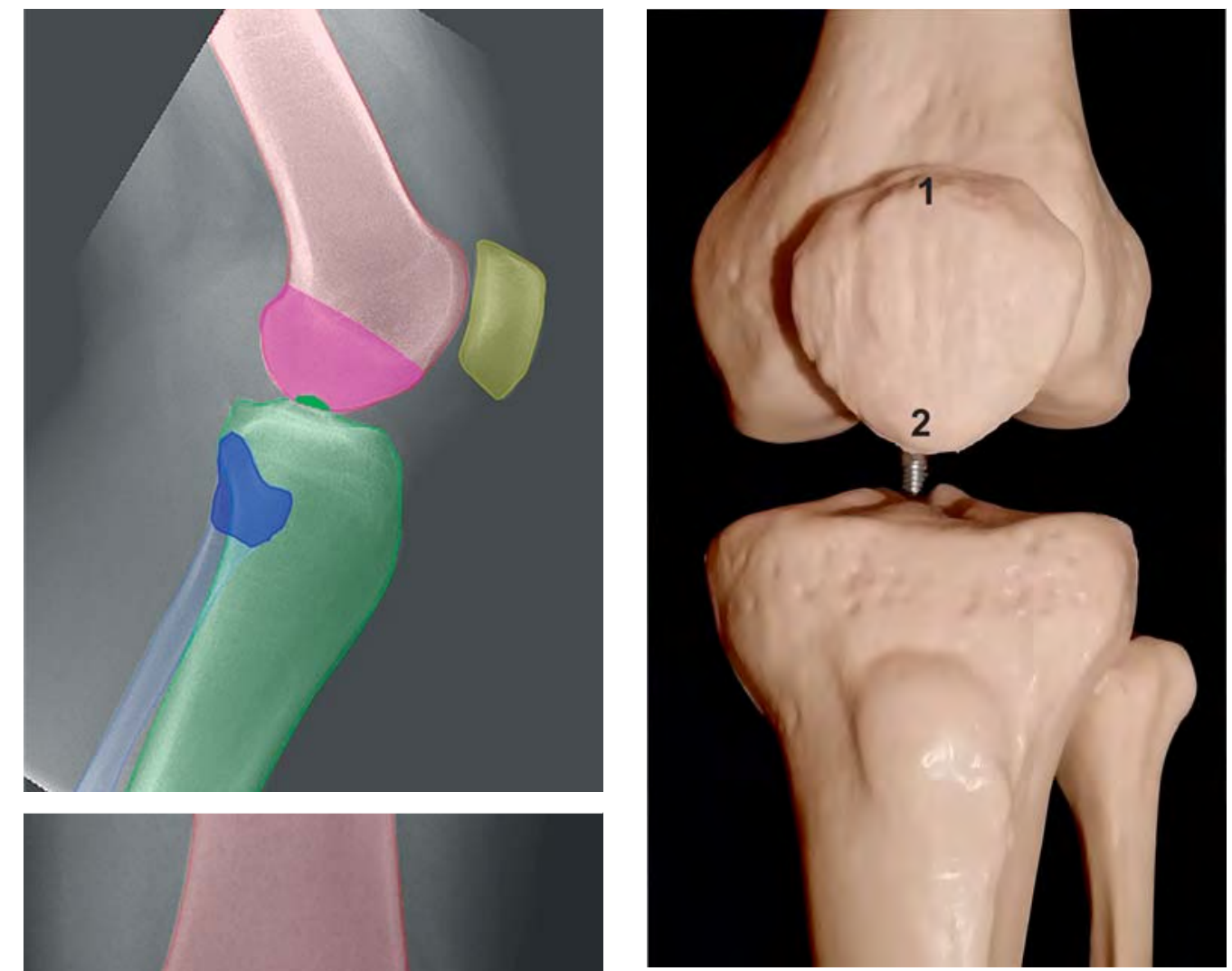

Abb. 3 Patella von oben.

1 Basis patellae.

2 Apex patellae.

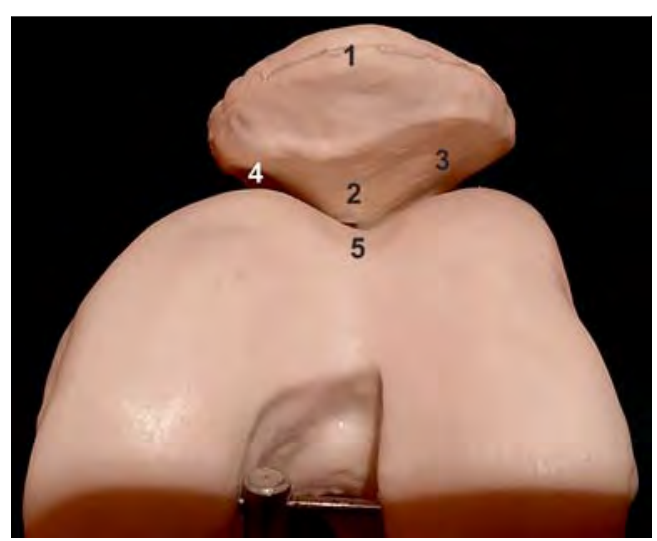

Abb.4 Patella von vorne.

1 Basis patellae.

2 Patellafirst.

3 Laterale Gelenkfläche.

4 Mediale Gelenkfläche.

5 Facies patellaris. 


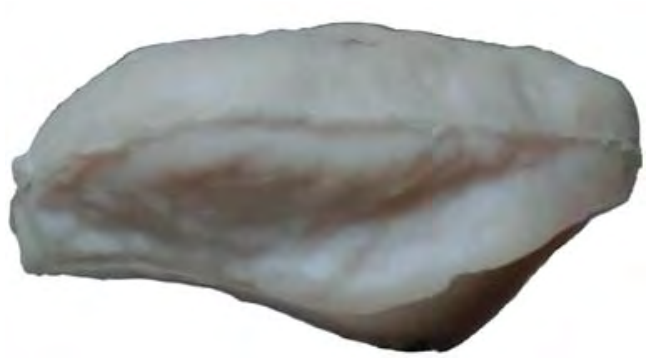

Abb.5 Patella einzeln von vorne.

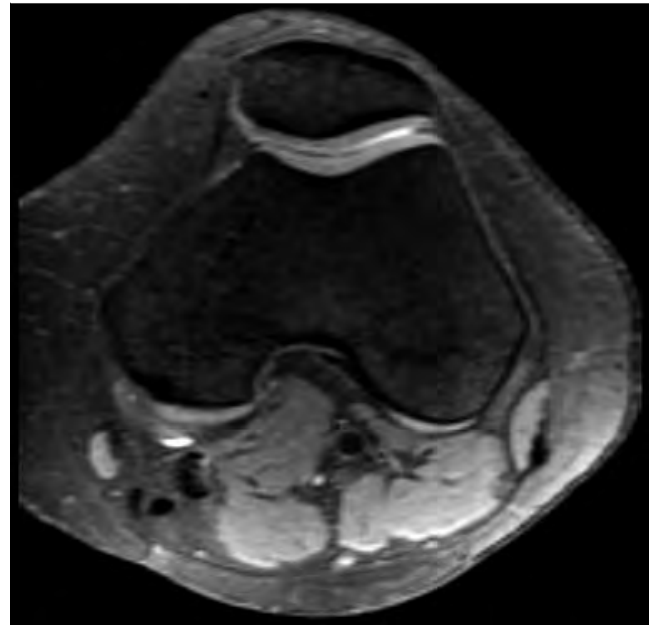

Abb. 6 Axialer MRT-Schnitt: Sehr gut zu sehen sind das Gleitlager und die kräftige Knorpelschicht, auf der die Patella gleitet.

\section{Hintergrundwissen Patella}

- Die Patella ist das größte Sesambeim.

- Die Patella fungiert als „Umlenkrolle“.

Die Patella ist in die Sehne des M. quadriceps eingebettet.

Es gibt 4 Muskelanteile des M. quadriceps: Rectus femoris

Vastus medialis

Vastus lateralis

Vastus intermedius

\section{Konventionelle Bildgebung der Patella}

Für die Diagnose dieser Veränderungen ist die konventionelle Bildgebung ein wesentlicher Bestandteil, wobei nicht alle Veränderungen pathologischen Wert haben. Es lassen sich bei der konventionellen Bildgebung sowohl die Form der Patella, die Lage und Beziehung zum Gleitlager als auch eine Lateralisation bis hin zur Luxation und Verkippung der Patella darstellen. Bei gut eingestellten Aufnahmen können anhand anatomischer Landmarks (z. B. mediale und laterale Patellafacette, Patellafirst) wichtige Messungen durchgeführt werden, die eine Klassifizierung erlauben und einen Hinweis auf die entsprechende Thera-

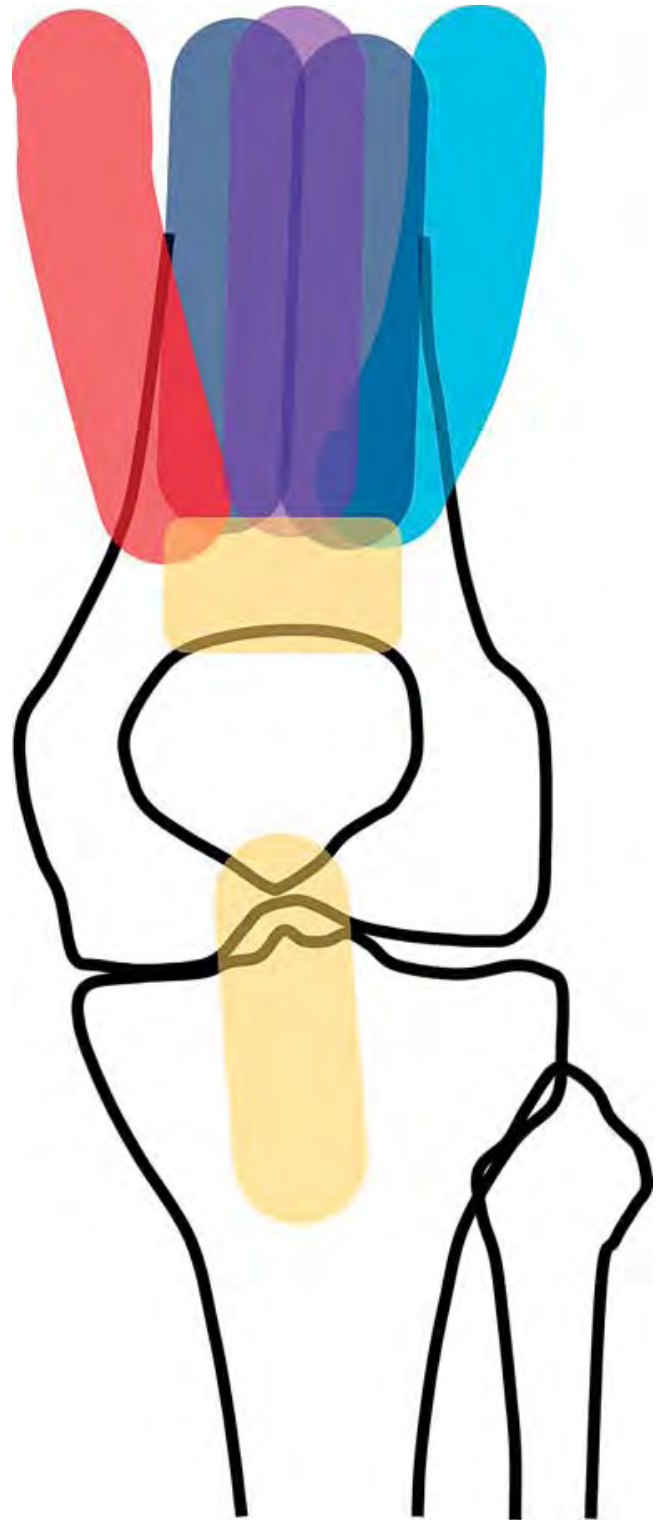

Abb.7 Skizze Bandsituation. Rectus femoris: violett, Vastus intermedius: grau, Vastus lateralis: hellblau, Vastus medialis: rot. pie geben. So kann z.B. die Verkippung der Patella in der horizontalen Ebene, der sog. Patella-TiltWinkel gemessen werden. Hier gibt es im Wesentlichen 2 Methoden:

\section{$\checkmark$ nach Laurin \\ nach Grelsamer}

Bei der konventionellen Bildgebung lassen sich sowohl die Form der Patella, die Lage und Beziehung zum Gleitlager als auch eine Lateralisation bis hin zur Luxation und Verkippung der Patella darstellen. 


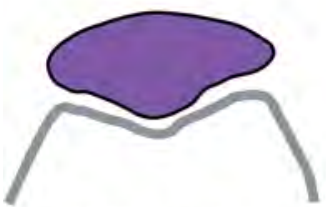

Wiberg 1

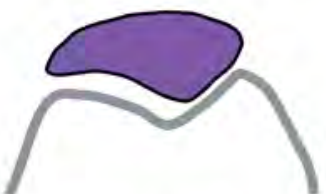

Wiberg 2

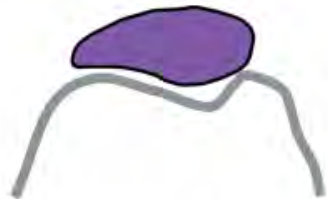

Wiberg 3

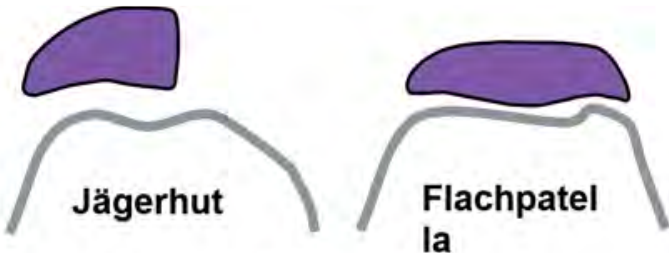

Wiberg 4

Abb. 8 Schema nach Wiberg.

Abb.9 Schema Patella partita.

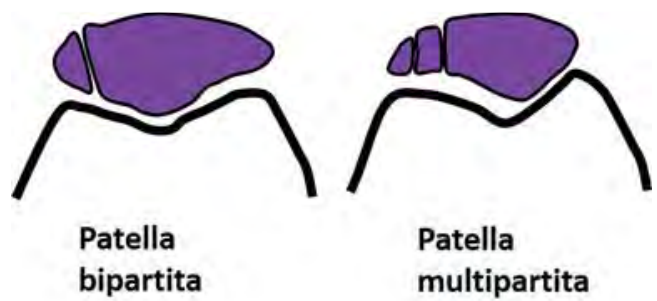

Abb.10 Patella-Tilt-Winkel nach Laurin.

Abb.11 Patella-Tilt-Winkel nach Grelsamer.

Abb.12 Laterale Patellaverschiebung.
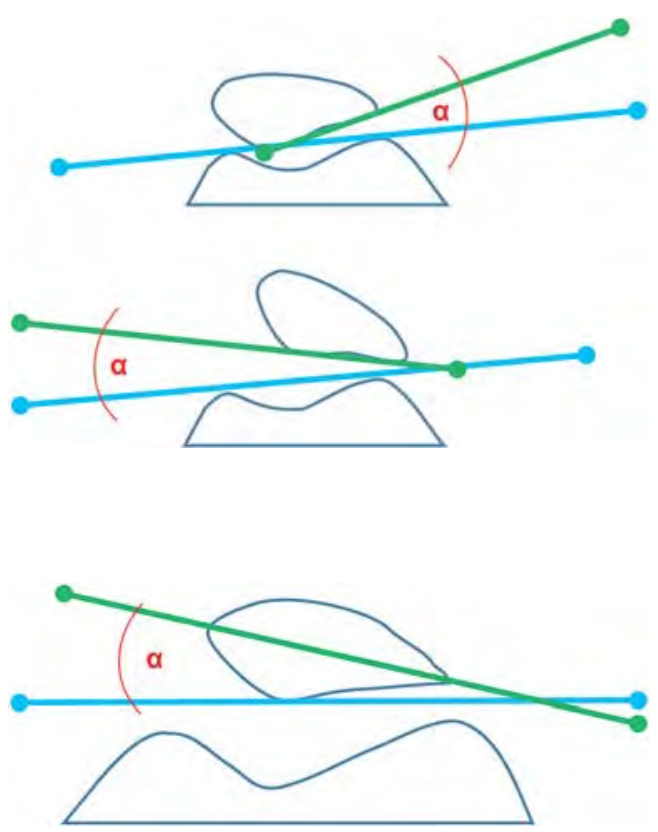

\section{Messung des Patella-Tilt-Winkels}

Bei den Methoden nach Laurin und Grelsamer ist eine exakte Einstellung essenziell für die Zuverlässigkeit der gemessenen Werte.

Methode nach Laurin. Bei der Bestimmung des lateralen patellofemoralen Winkels nach Laurin wird eine Linie durch die höchsten Punkte der Femurkondylen gelegt. Eine 2. Linie entlang der lateralen Patellafacette. Der dadurch entstehende Winkel ist beim Gesunden nach lateral geöffnet. Ist der Winkel nach medial geöffnet oder die Linien parallel, weist das auf eine pathologische Fehlstellung hin ( $\bullet$ Abb. 10).

Methode nach Grelsamer. Der Patella-Tilt-Winkel nach Grelsamer wird gemessen, indem man eine Linie durch das laterale und mediale Ende der Patellafacetten legt. In Verbindung mit der Horizontalen entsteht ein nach medial geöffneter Winkel ( $\triangle$ Abb.11). Ist der Winkel größer als $5^{\circ}$, gilt die Fehlstellung als pathologisch.

Laterale Patellaverschiebung. Ebenso kann z.B. auch eine Lateralisierung der Patella einfach ausgemessen werden. Hierzu werden die höchsten Punkte der Femurkondylen verbunden. Senkrecht dazu wird eine Hilfslinie durch den tiefsten Punkt des Patellafirsts und durch den tiefsten Punkt des Sulcus trochlearis gelegt. Der Abstand zwischen den Linien gibt die laterale Verschiebung der Patella an und sollte nicht mehr als $2 \mathrm{~mm}$ betragen. Auch hier kann eine Messung nur bei gut eingestellten Bildern zuverlässig erfolgen ( $\bullet$ Abb. 12).

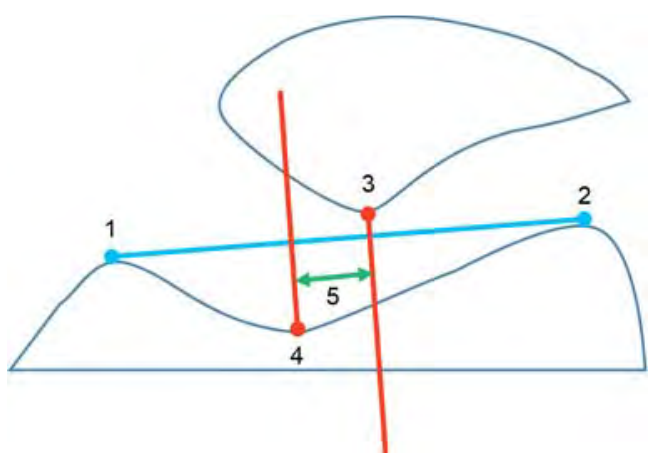

Neben der Standardaufnahme Knie in 2 Ebenen sind Spezialaufnahmen der Patella notwendig. In diesem Beitrag werden die Patella tangential, Patella Defilée und Patella nach Merchant vorgestellt ( Tab.1 u. Tab.2).

Der Patella-Tilt-Winkel kann nach den Methoden nach Laurin und Grelsamer gemessen werden. 
Tab. 1 Spezialaufnahmen der Patella.

\begin{tabular}{|l|l|}
\hline Aufnahme & Indikationen \\
\hline Patella tangential & Praktur \\
& pegenerative Veränderungen \\
& position im Gleitlager \\
\hline Patella Defilée & Patellaluxation (in Abhängigkeit von der Kniebeugung) \\
\hline Patella nach Merchant & pathologische Luxation \\
\hline
\end{tabular}

Tab. 2 Technische Einstellparameter für die Belichtung für alle angeführten Aufnahmen.

\begin{tabular}{|l|l|l|l|l|l|l|l|}
\hline Format & Empfindlichkeitsklasse & Raster & Fokus-Detektor-Abstand $(\mathbf{c m})$ & Fokus & kV & mAs & Belichtungskammer \\
\hline 18/24 hoch & 400 & ohne & 105 & klein & 60 & 5 & ohne \\
\hline Leitlinien & $\begin{array}{l}400 \\
<5 \mu \mathrm{Gy}\end{array}$ & ohne & 105 & $\leq 1,3$ & $60-75$ & ohne \\
\hline
\end{tabular}

\section{Grundsätzliches zur Lagerung}

Für alle aufgeführten Einstellungen ist eine exakte Lagerung des Patienten unabdingbar. Der wichtigste Punkt ist hier eine achsgerechte Lagerung, das bedeutet, auch die Beinachse sollte gerade sein $(\bullet$ Abb. 13). Eine andere Lagerung $(\bullet$ Abb. 14-16) wirkt sich erheblich auf die Qualität der Aufnahmen aus. Oft sind dann die Bilder eingeschränkt oder gar nicht beurteilbar und die Aufnahme muss wiederholt werden.

Eine exakte, achsgerechte Lagerung des Patienten ist für eine gute Qualität der Aufnahmen absolut notwendig.

\section{Patella tangential}

Für die Patella tangential gibt es 2 verschiedene Einstellmöglichkeiten. Bei beiden ist es wichtig, den Patienten achsgerecht zu lagern und die Beinachse ebenfalls gerade zu lagern. Zu beachten ist hier, dass bei einer Patellaquerfraktur ( $\odot$ Abb.17) keine tangentiale Patella angefertigt werden darf.

Achtung: Bei einer Patellaquerfraktur darf keine tangentiale Patellaaufnahme angefertigt werden!

\section{Bauchlage}

\section{$\nabla$}

Der Patient liegt in Bauchlage auf dem Untersuchungstisch. Der Unterschenkel wird über den Oberschenkel gebeugt, wenn nötig zieht der Patient mit einem Band den Unterschenkel nach hinten. Dabei ist darauf zu achten, dass der Oberschenkel auf dem Tisch aufliegt. Die Zentrierung erfolgt auf die Patellaspitze und sollte durch den Femoropatellarspalt führen. Dies kann mit einer Röhrenkippung nach kranial verbunden sein ( $\triangle$ Abb. 18). Wenn mit dem Band gearbeitet wird, sollte darauf geachtet werden, das Band über die Schulter der Gegenseite zu nehmen, da so Ober-

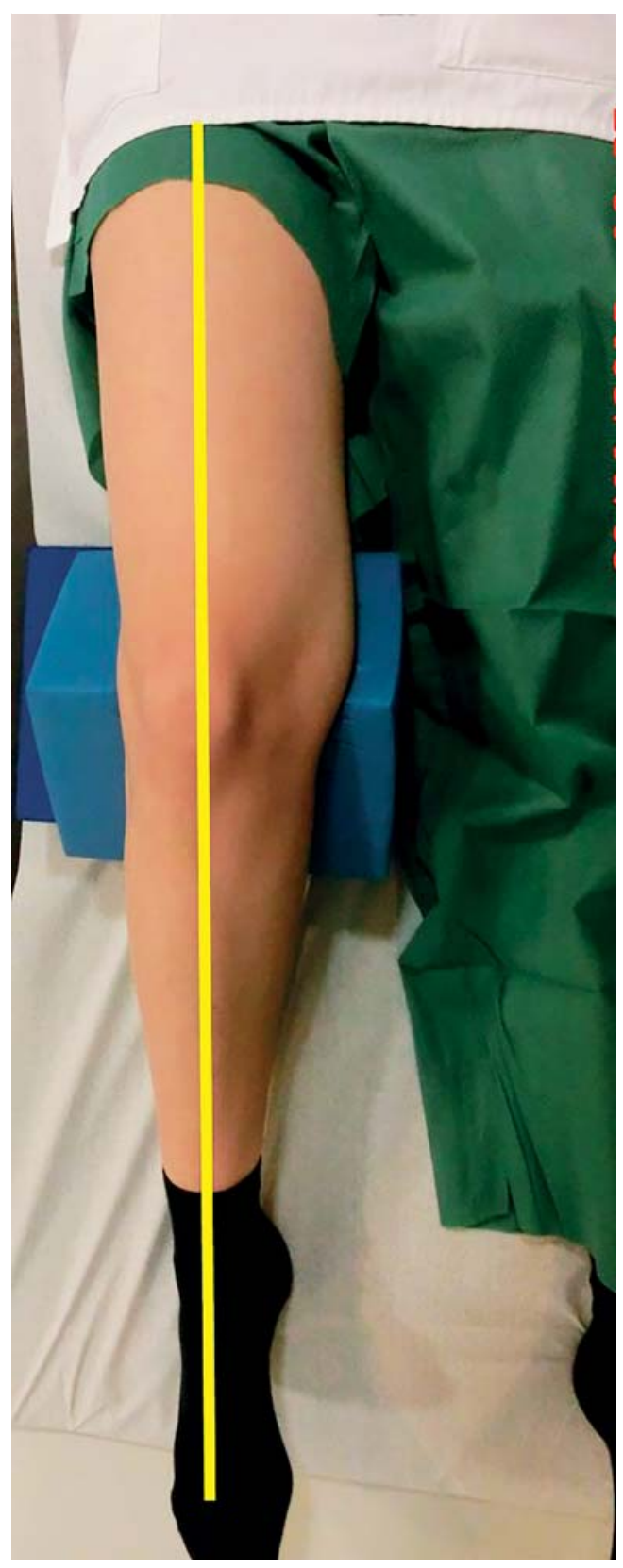

Abb.13 Patient achsgerecht gelagert, Beinachse gerade. 
Abb. 14 Beinachse verdreht.

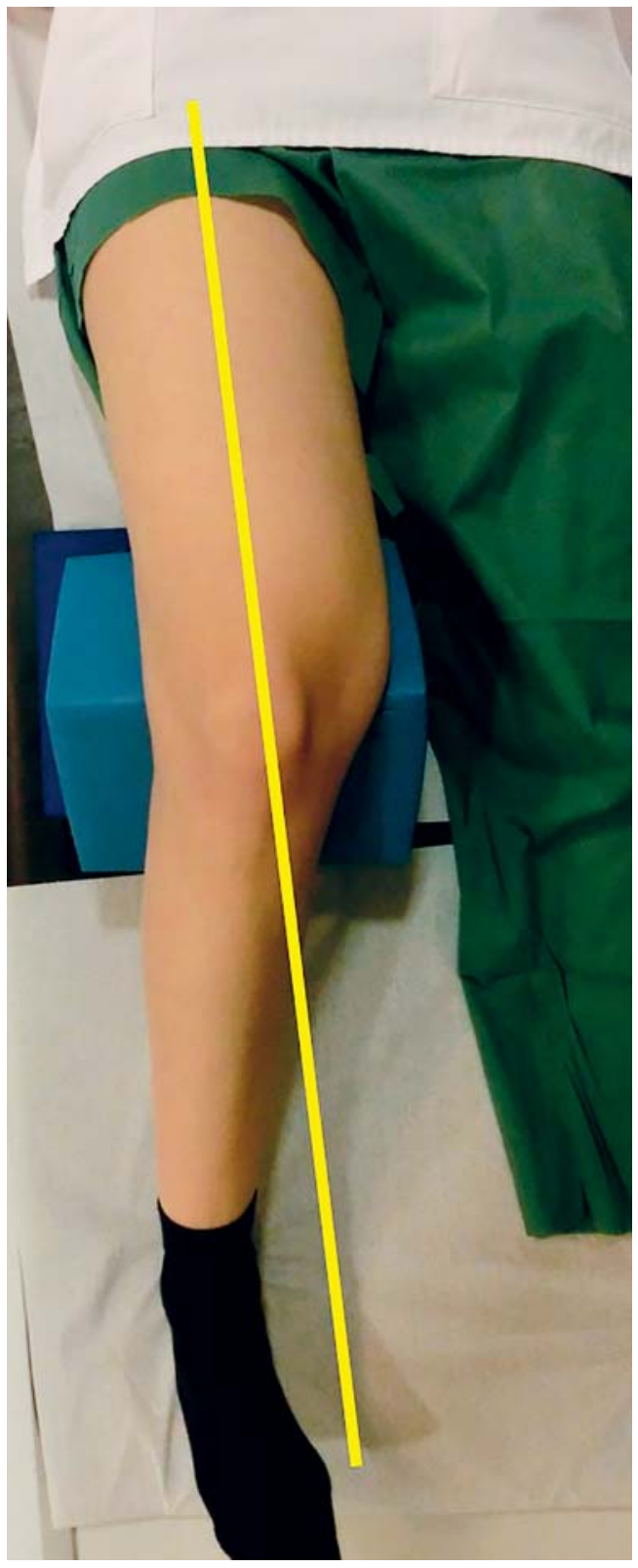

und Unterschenkel besser übereinander gelagert werden können und eine Verkippung nach lateral vermieden werden kann ( $\bullet$ Abb.19 u. $\bullet$ Abb.20).

Aus Sicht des Strahlenschutzes ist diese Aufnahme der folgenden Aufnahme in Rückenlage vorzuziehen. In Abb.19 u. Abb.20 wurde der obligatorische Strahlenschutz für die Bilddokumentation entfernt, um die Stellung von Oberund Unterschenkel besser darzustellen.

Aus Sicht des Strahlenschutzes ist die Lagerung des Patienten in Bauchlage für die Aufnahme der Patella tangential zu bevorzugen.

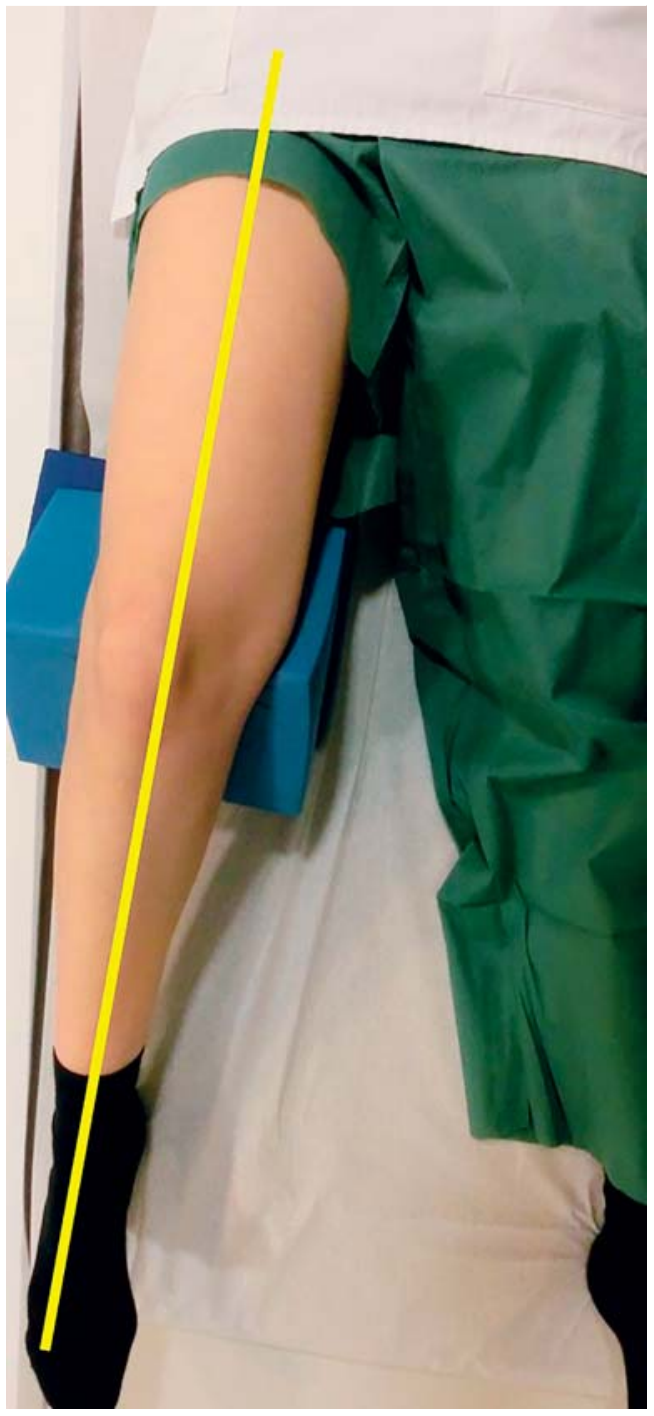

Abb.15 Beinachse nach lateral.

\section{Rückenlage}

Der Patient liegt auf dem Tisch. Das zu untersuchende Bein wird angestellt. Der Patient hält die Kassette senkrecht zum Tisch ( Abb.21). Der Zentralstrahl wird auch hier nach dem Retropatellarspalt auf die Patellaspitze ausgerichtet. Auch bei dieser Aufnahme ist es wichtig, die Beinachse gerade auszurichten. Diese Aufnahme im Sitzen durchzuführen, ist aus Strahlenschutzaspekten obsolet. Um den Retropatellarspalt zu finden, tastet man die Patellaspitze und die Patellabasis. Durch diese beiden Punkte sollte der Zentralstrahl verlaufen ( $\bullet$ Abb.22). 


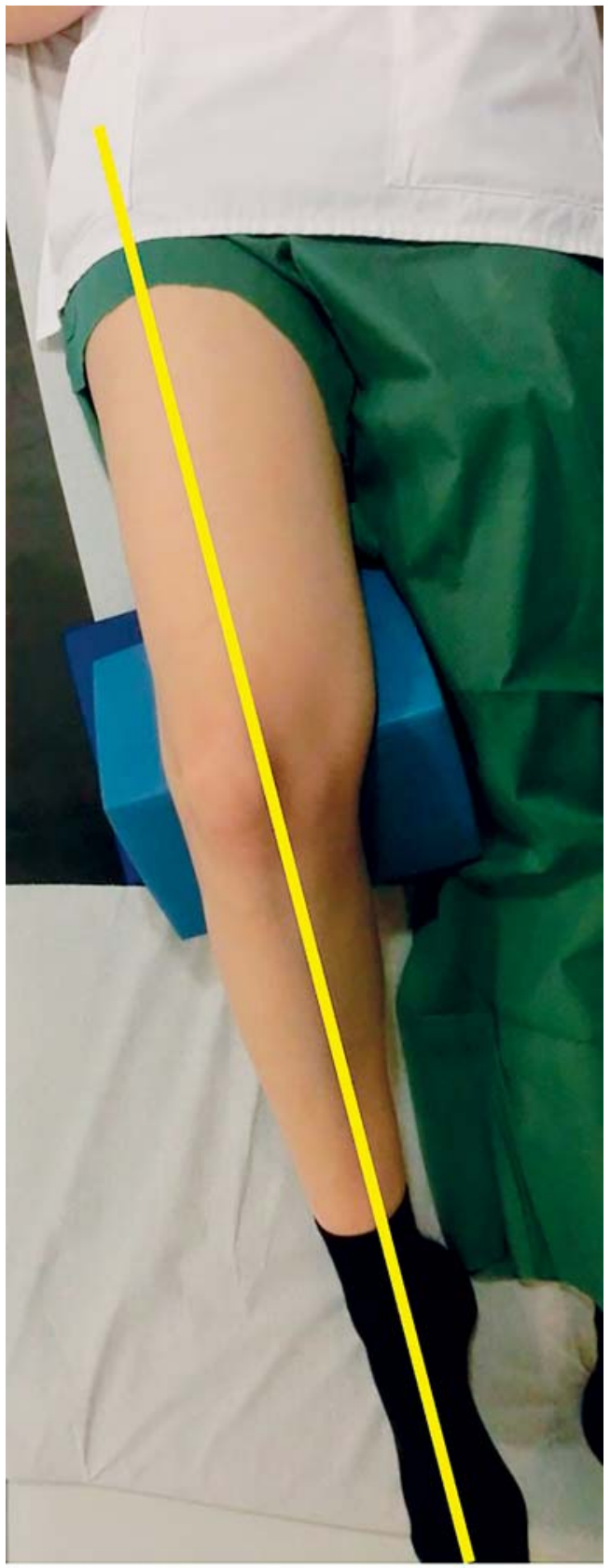

Abb.16 Beinachse nach medial.

\section{Kriterien einer gut eingestellten Aufnahme $\nabla$}

Auf der Aufnahme müssen die gesamte Patella, der Retropatellarspalt und das femoropatellare Gleitlager gut beurteilbar sein ( $\bullet$ Abb.23). Die Patellarückfläche ist strichförmig dargestellt. Ist das nicht der Fall, können weder exakte Messungen durchgeführt werden, noch können Veränderungen der Knochen und des Gelenkspalts beurteilt werden ( $\triangle$ Abb.24-27). Ein häufiger Fehler ist hier eine falsche Röhrenkippung oder eine falsche Ausrichtung der Beinachse, die eine gute Einsicht in den Retropatellarraum erlaubt.
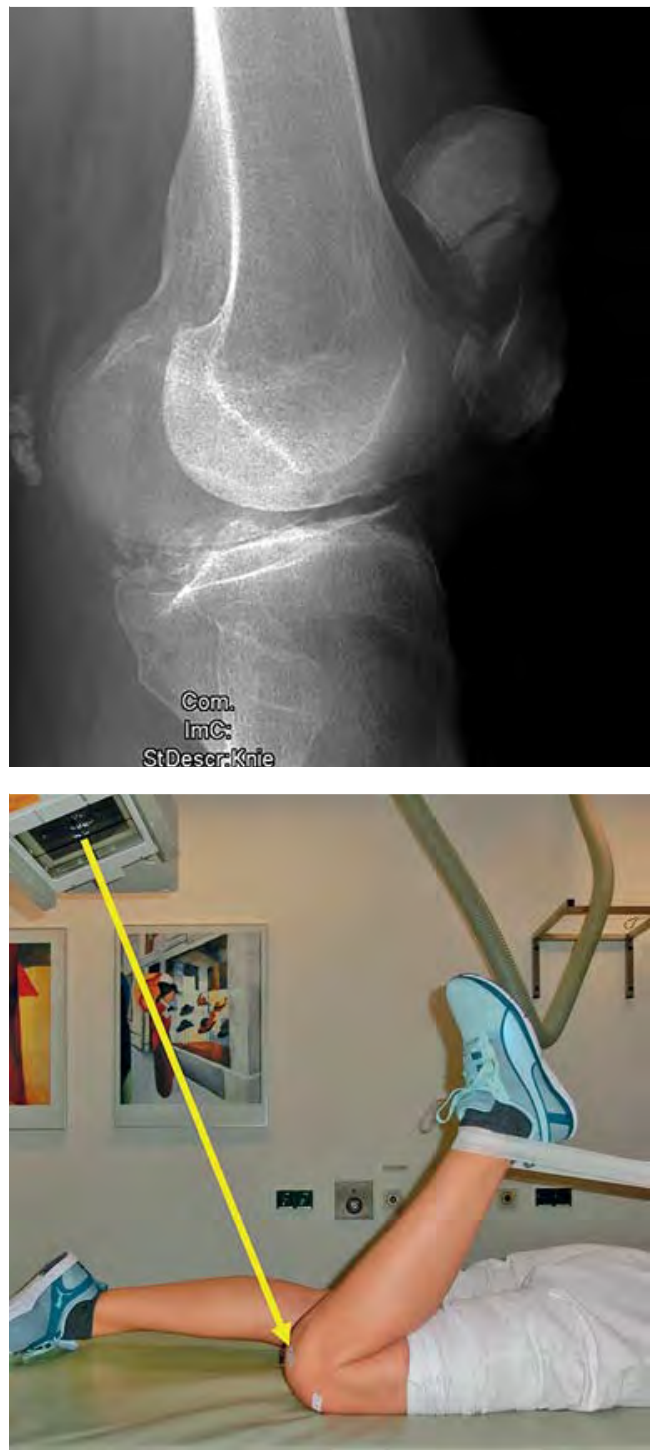

Abb. 18 Lagerung und Zentrierung Patella tangential in Bauchlage.

Bei einer schlecht eingestellten Patella tangential können weder exakte Messungen durchgeführt werden, noch können Veränderungen der Knochen und des Gelenkspalts beurteilt werden.

\section{Tipps zur Lagerung}

$\nabla$

Wenn vorhanden, sollte der Halteapparat für die Patella-Defilée-Aufnahme verwendet werden. Hierdurch wird sichergestellt, dass der Patient bequem und sicher gelagert werden kann, ohne dass Bewegungsartefakte entstehen. Steht kein Halteapparat zur Verfügung, muss der Patient unter Verwendung von Polstern und Lagerungshilfen unter dem Knie in die entsprechende Winkelposition gebracht werden.

Abb. 17 Röntgenbild einer Patellafraktur. 
Abb. 19 Lagerung Bauchlage, Oberschenkel und Unterschenkel nicht übereinander.
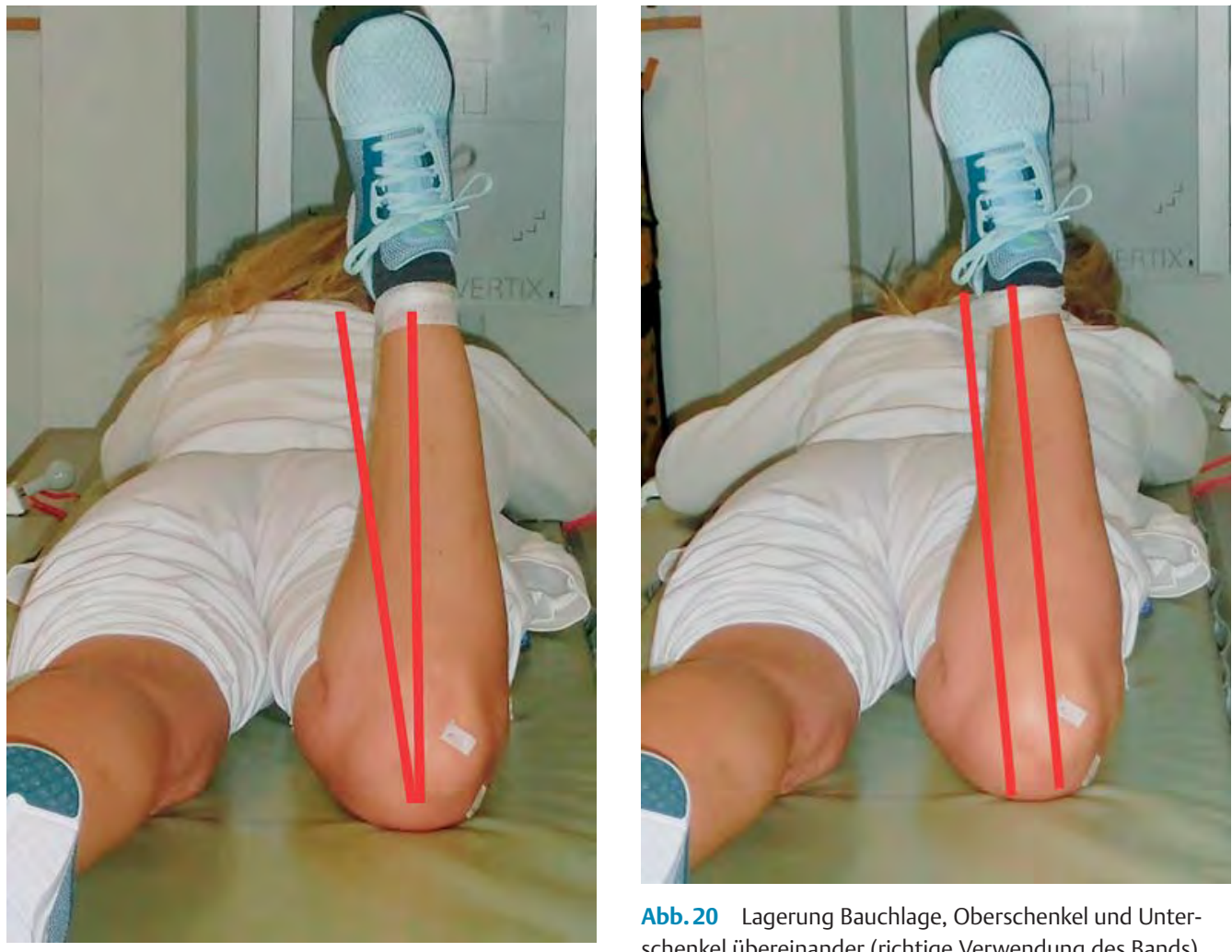

Abb. 20 Lagerung Bauchlage, Oberschenkel und Unterschenkel übereinander (richtige Verwendung des Bands).

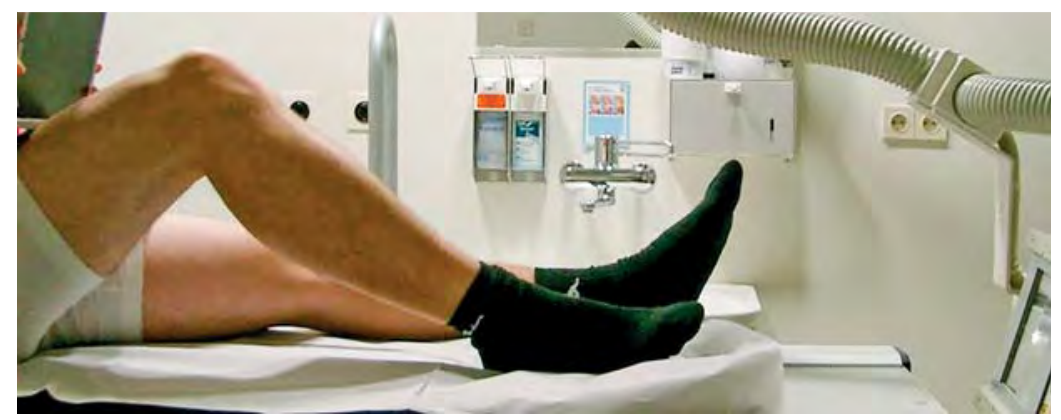

Abb.21 Lagerung Patella tangential in Rückenlage.

Abb.23 Röntgenbild gut eingestellte Patella tangential.

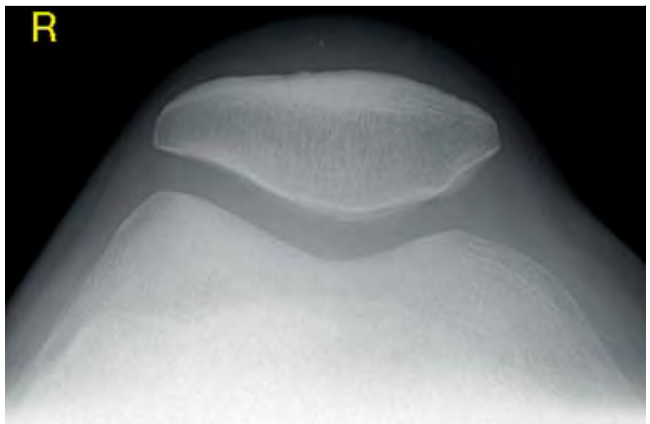

Abb.22 Der Zentralstrahl trifft genau den Retropatellarspalt.

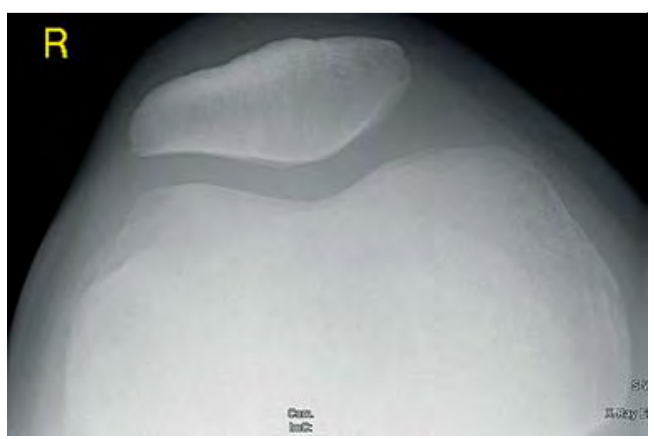

Abb.24 Röntgenbild gut eingestellte Patella tangential. 


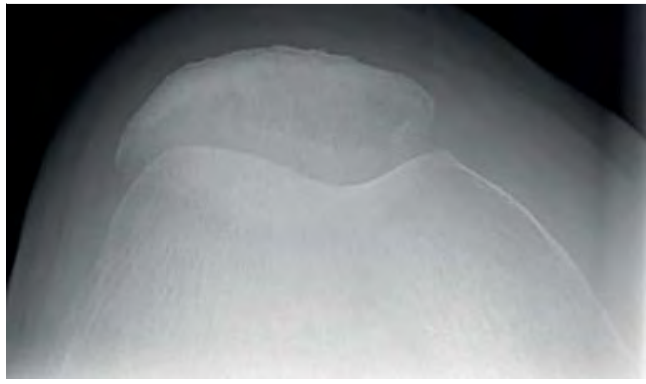

Abb.25 Röntgenbild schlecht eingestellte Patella tangential (Beinachse verdreht, falsche Röhrenkippung).

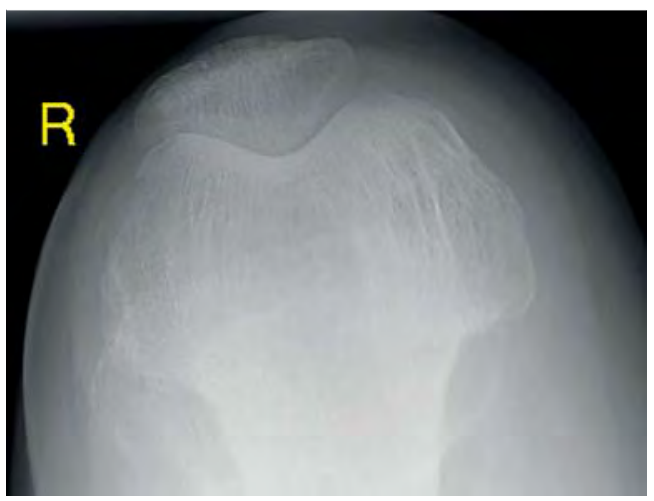

Abb.26 Röntgenbild schlecht eingestellte Patella tangential (Beinachse verdreht, falsche Röhrenkippung).

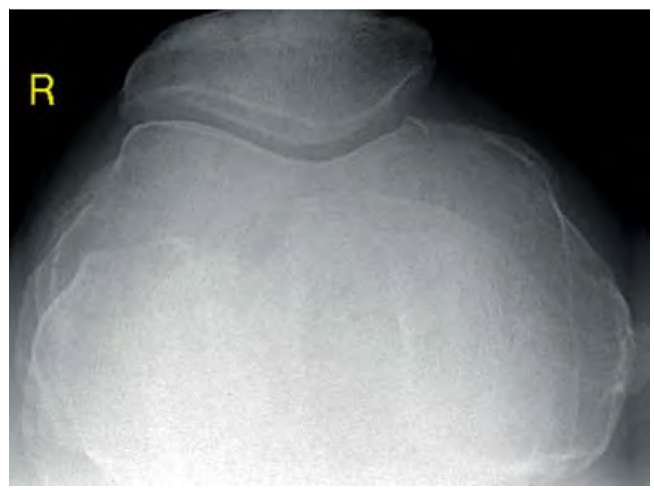

Abb.27 Röntgenbild schlecht eingestellte Patella tangential (falsche Röhrenkippung).

Tipps und Tricks:
Lagerung Patella tangential
> achsgerechte Lagerung des Patienten
- Lagerungshilfsmittel verwenden
- Retropatellarspalt einsehbar
- Röhrenkippung nach Patellarückfläche

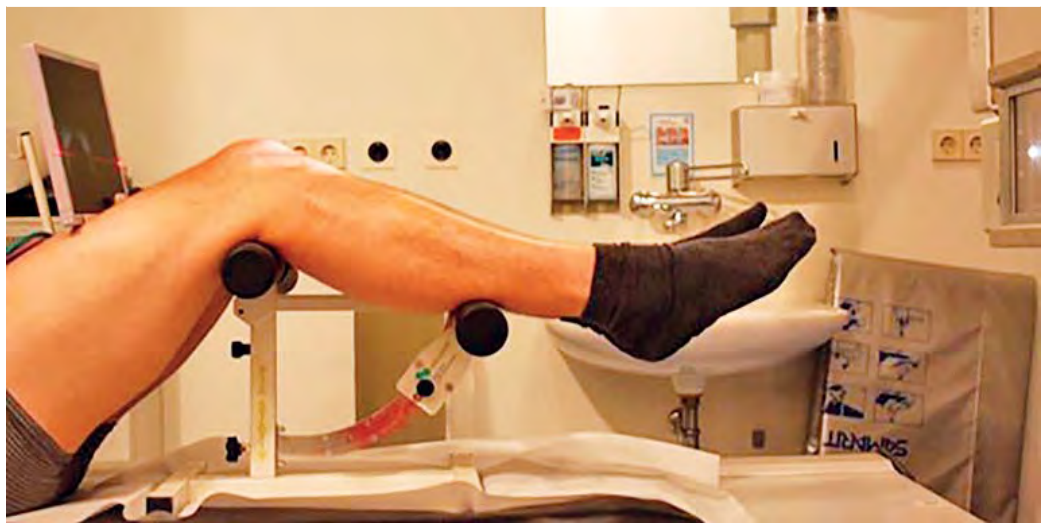

Abb. 28 Lagerung Patella Defilée mit Lagerungshilfe (Einstellung $30^{\circ}$ ).

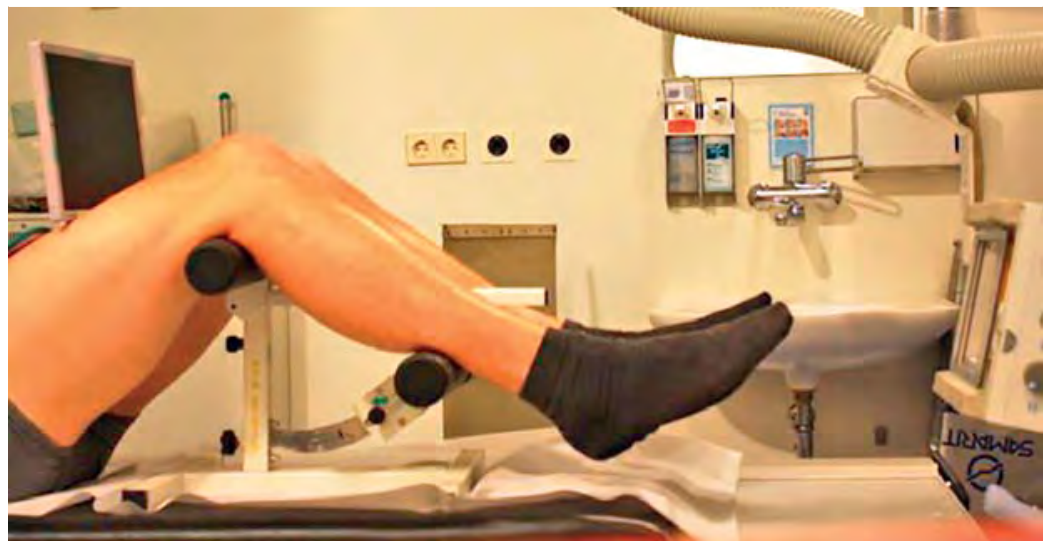

Abb. 29 Lagerung Patella Defilée mit Lagerungshilfe (Einstellung 60 ).

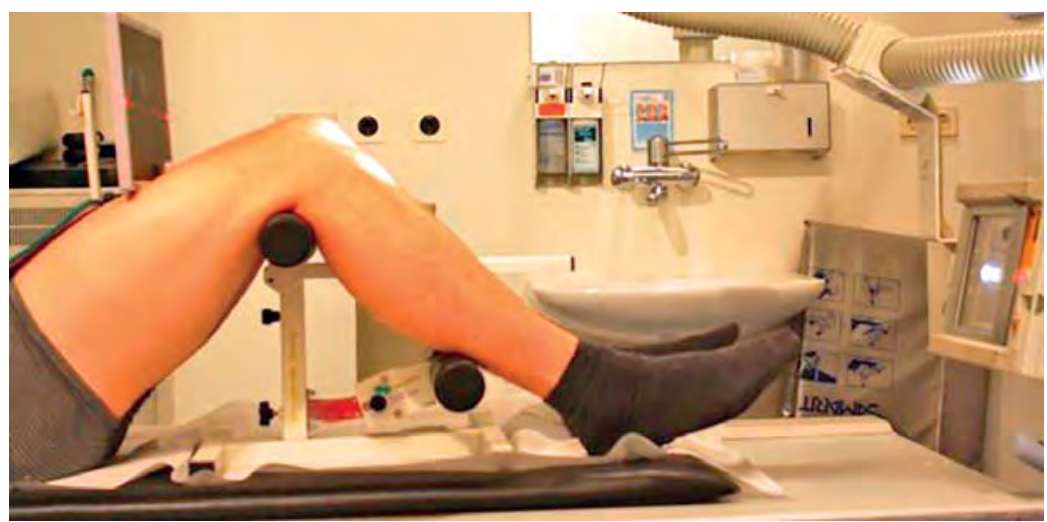

Abb.30 Lagerung Patella Defilée mit Lagerungshilfe (Einstellung 90²).

\section{Patella Defilée}

Zur Darstellung einer Patellaluxation unter Einfluss der Kniebeugung werden Patellaaufnahmen nach Defilée angefertigt. Für diese Aufnahmen liegt der Patient auf dem Rücken, die Medianeben und die Beinachse müssen wieder gerade sein. Es werden 3 Aufnahmen der Patella in $30^{\circ}-, 60^{\circ}$ - und $90^{\circ}$-Kniebeugung angefertigt (० Abb. 28-30). 
Abb. 31 Gut eingestellte Patella Defilée $30^{\circ}$.

Abb.32 Gut eingestellte Patella Defilée $60^{\circ}$.

Abb.33 Gut eingestellte Patella Defilée $90^{\circ}$

Abb.34 Schlecht eingestellte Patella Defilée $30^{\circ}$

Abb.35 Schlecht eingestellte Patella Defilée $60^{\circ}$
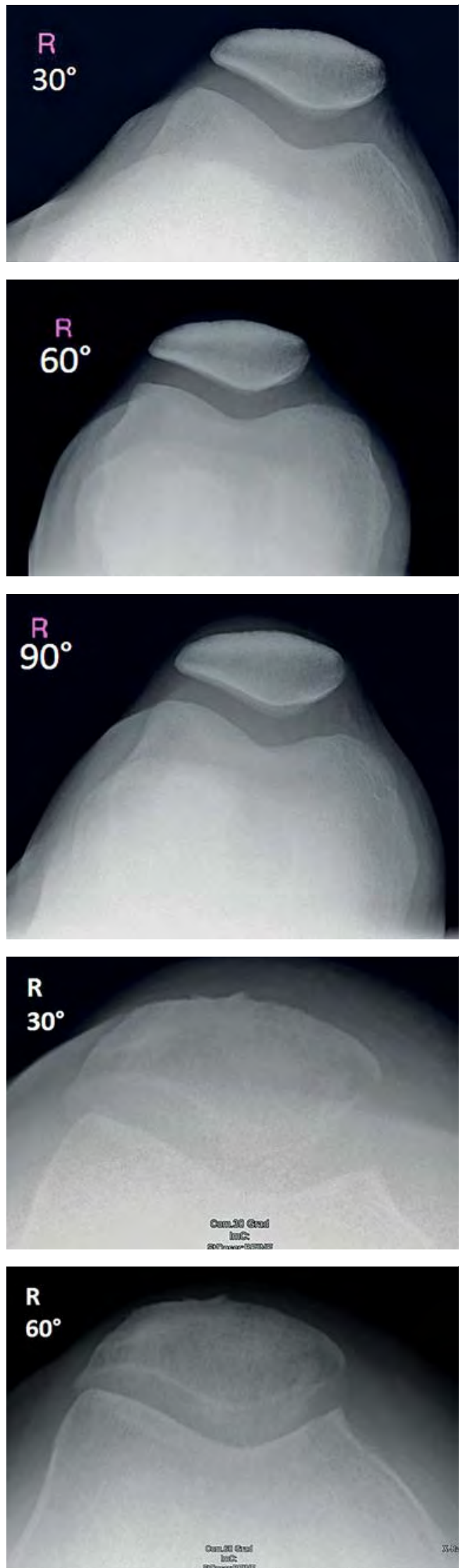

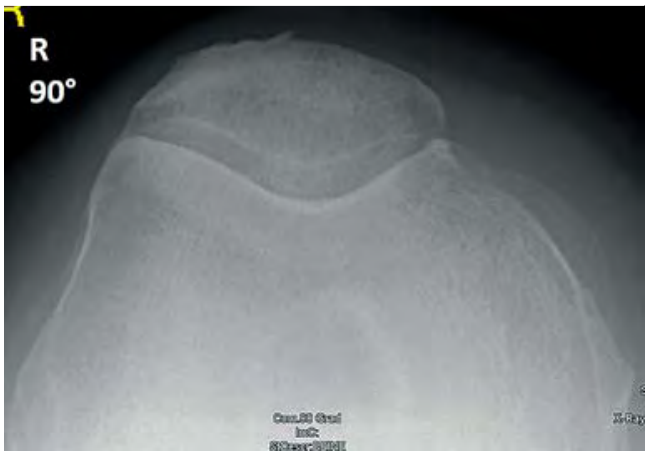

Abb.36 Schlecht eingestellte Patella Defilée $90^{\circ}$.

Patellaaufnahmen nach Defilée werden zur Darstellung einer Patellaluxation angefertigt.

\section{Kriterien einer gut eingestellten Aufnahme \\ $\nabla$}

Das Kriterium der guten Aufnahme ist - wie bei der Patella tangential - eine gute Einsicht in den Retropatellarspalt und eine gute Beurteilbarkeit der Patellarückfläche. Eine Lateralisierung der Patella ist in Abb.31-33 nicht erkennbar. Eine falsche Kippung des Zentralstrahls führt zu nicht beurteilbaren Ergebnissen, wie in Abb.34-36 zu sehen ist. Hier ist der Retropatellarspalt nicht einsehbar.

\section{Tipps zur Lagerung \\ $\nabla$}

Um reproduzierbare Ergebnisse zu erreichen, ist es sinnvoll, ein Hilfsmittel zu verwenden. Mithilfe des in Abb. 28-30 dargestellten Geräts ist es möglich, auch bei Verlaufsaufnahmen vergleichbare Bilder zu erstellen.

\section{Tipps und Tricks: Lagerung Patella Defilée \\ achsgerechte Lagerung des Patienten \\ - Lagerungshilfsmittel verwenden \\ - Retropatellarspalt einsehbar \\ - Röhrenkippung nach Patellarückfläche \\ - Aufnahmen in $30^{\circ}$-, $60^{\circ}$-, $90^{\circ}$-Kniebeugung}




\section{Patella nach Merchant}

Die Patella nach Merchant ist eine Möglichkeit, die Patellaluxation darzustellen bzw. auszuschließen. Dazu ist es erforderlich, die Aufnahme im Seitenvergleich anzufertigen. Voraussetzung hier ist ein Anbau an den Röntgentisch in Form einer Platte, die mit Scharnieren befestigt ist. Über eine Stange ist die Winkeleinstellung mittels Tischbewegung realisiert. Zusätzlich ist noch eine Kassettenhalterung integriert ( Abb.37).

Der Patient sitzt hier an der Tischkante und lässt die Beine auf der Zusatzplatte, auch liebevoll Pizzablech genannt, aufliegen. Der Tisch wird soweit verschoben, dass ein $45^{\circ}$-Winkel zwischen Oberschenkel und Unterschenkel entsteht. Der Zentralstrahl wird kraniokaudal auf den Retropatellarspalt gerichtet.

Die Patella nach Merchant ist eine Möglichkeit, die Patellaluxation darzustellen bzw. auszuschließen. Die Aufnahme muss im Seitenvergleich angefertigt werden.

\section{Kriterien einer gut eingestellten Aufnahme \\ $\nabla$}

Das Kriterium der guten Aufnahme ist - wie bei der Patella tangential - eine gute Einsicht in den Retropatellarspalt und eine gute Beurteilbarkeit der Patellarückfläche.

\section{Tipps zur Lagerung}

$\nabla$

Das Ergebnis kann verfälscht werden, wenn der Patient die Beine zusammendrückt. Dadurch wird unter anderem der Vastus medialis angespannt. Er zieht die Patella nach medial und kranial und kann so eine Pathologie verschleiern. Sinnvoll ist es, dem Patienten die Oberschenkel mit einem Band zusammenzubinden, um die Beinachse gerade zustellen ( $\bullet$ Abb.38). Dieses Vorgehen entspannt den Vastus medialis, und eine Patellaluxation kann zur Darstellung kommen.

\footnotetext{
Tipps und Tricks:

Lagerung Patella nach Merchant

- achsgerechte Lagerung des Patienten

- Lagerungshilfsmittel verwenden

- Retropatellarspalt einsehbar

- Röhrenkippung nach Patellarückfläche

- Aufnahmen in $45^{\circ}$-Kniebeugung

beine mit einem Band zusammenbinden
}

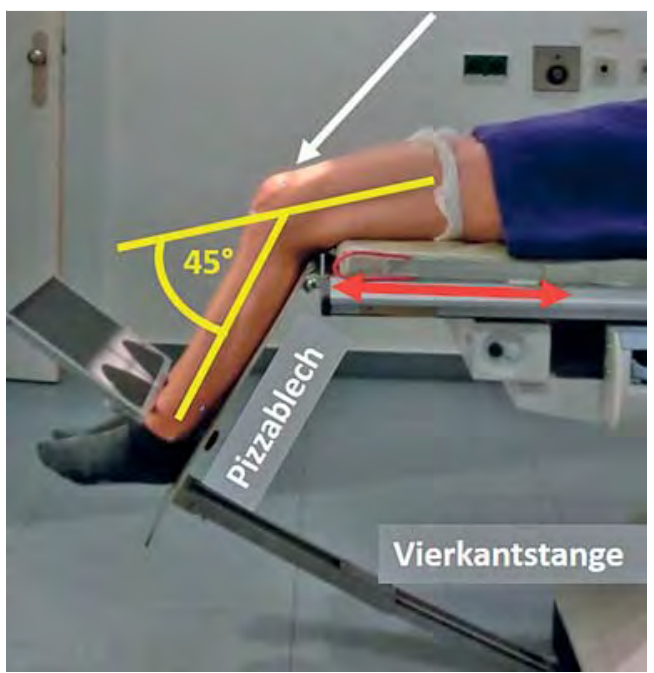

Abb. 37 Lagerung Patella nach Merchant.

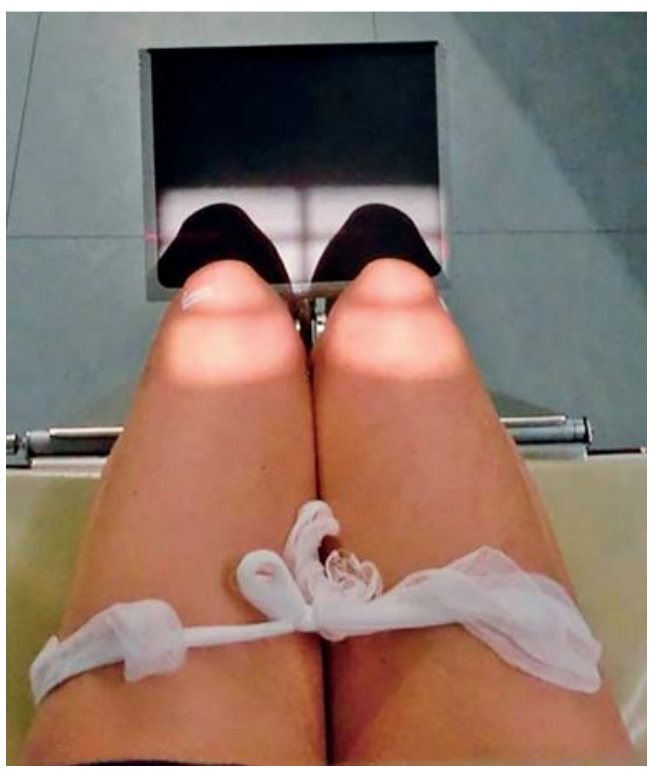

Abb.38 Zentrierung und Einblendung Patella nach Merchant inklusive Band um die Oberschenkel.

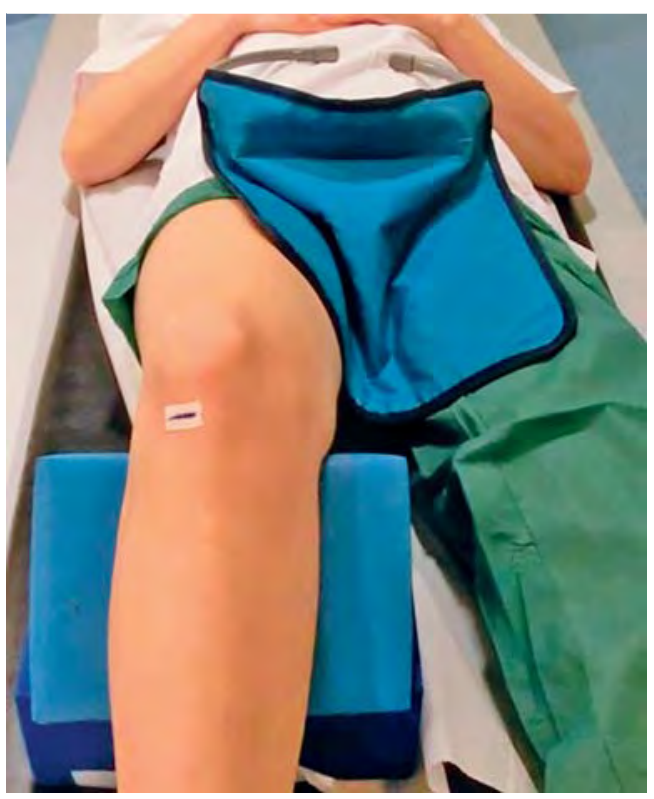

Abb. 39 Lagerung Patella tangential in Rückenlage mit richtig angelegtem Strahlenschutz. 


\section{Strahlenschutz}

Wie bei allen Röntgenuntersuchungen ist auch bei den hier vorgestellten Aufnahmen auf den Strahlenschutz zu achten. Die passende Belichtung, der entsprechende Film-Detektor-Abstand und eine möglichst exakte Einblendung sind hier zu beachten. Bei Patientinnen ist die Frage nach einer möglichen Schwangerschaft zu stellen und im Zweifelsfall ein Schwangerschaftstest zu machen. Bei bestehender Schwangerschaft ist die Indikation äußerst streng zu stellen. Bei Aufnahmen die von fußwärts durchgeführt werden, sollte die Strahlenschutzschürze immer zwischen die Beine gelegt werden ( $\bullet$ Abb.39), damit die von kaudal kommende Streustrahlung auf die Gonaden abgeschirmt wird. Wenn möglich sollte immer eine Strahlengangrichtung gewählt werden, die vom Körper weggeht.

\section{Abstract}

It can happen at work, sports, or games. A sudden pain in the knee, a fall, or the like. One of the possible reasons is an injury or involvement of the patella. To be certain, usually specific radiographs are required. In this article patella tangential view, patella sunrise view (axial projection in $30^{\circ}-60^{\circ}-90^{\circ}$ flexion of the knee), and Merchant technique are depicted. The accurate adjustment and mistakes as well as their elimination are discussed.

Bei Aufnahmen die von fußwärts durchgeführt werden, sollte die Strahlenschutzschürze immer zwischen die Beine gelegt werden.

\section{Kernaussagen}

- Eine gute, achsgerechte Lagerung des Patienten ist entscheidend.

- Eine falsche Lagerung verfälscht das Ergebnis; die Patella ist nur eingeschränkt oder nicht beurteilbar.

- Der Retropatellarspalt muss einsehbar sein.

- Zur Lagerung sollten Lagerungshilfsmittel verwendet werden.

- Der Zentralstrahl ist nach der Patellarückfläche zu kippen.

Der Strahlenschutz muss beachtet werden.

- Die Patella tangential wird bei Verdacht auf Verletzung und Degeneration angefertigt

Die Patella Defilée wird bei Verdacht auf Luxation in $30^{\circ}$-, $60^{\circ}$ - und $90^{\circ}$-Kniebeugung aufgenommen

Die Patella nach Merchant wird bei Verdacht auf Luxation im Seitenvergleich in $45^{\circ}$-Kniebeugung aufgenommen.

- Die Zentrierung erfolgt $1 \mathrm{~cm}$ unterhalb der Patellaspitze.

- Eine Einblendung auf das Objekt ist notwendig. 


\section{Literatur}

1 AWMF online. S1-Leitlinie Patellafraktur, AWMFRegister-Nr. 012/017. Im Internet: http://www. awmf.org/uploads/tx_szleitlinien/012-0171_Patellafraktur_2014-06.pdf; Stand: 10.4.2016

2 Bernau A. Orthopädisch-Traumatologische Röntgendiagnostik: Einstelltechnik. 4. Aufl. München: Elsevier; 2004

3 Fößler A, Pfeffer R. Konventionelle Röntgendiagnostik: Einstelltechnik und Röntgenanatomie. München: Elsevier; 2005

4 Waldt S, Eiber M, Wörtler K. Messverfahren und Klassifikationen in der muskuloskelettalen Radiologie. Stuttgart: Thieme; 2011

\section{Über den Autor}

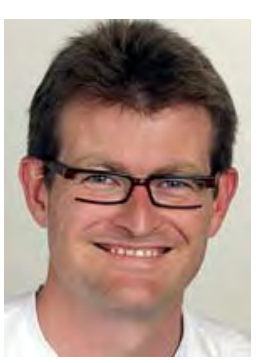

Claus Becker, Leitender MTRA

Jahrgang 1971. $1982-1993$

Schulausbildung in Metten. 1994-1997 Ausbildung an der MTRA-Schule München Großhadern. Seit 1997 MTRA am Institut für Röntgendiagnostik am Uniklinikum Regensburg. Seit 2015 Vorstandsvorsitzender der VMTB

\section{Korrespondenzadresse}

Claus Becker

Institut für Röntgendiagnostik

Uniklinikum Regensburg

Franz-Josef-Strauss-Allee 11

93053 Regensburg

Deutschland

E-Mail: Claus.Becker@UKR.de

Interessenkonflikt: Es besteht kein Interessenkonflikt.

\section{Radiobonus}

Sie können eines von 3 Exemplaren des Bandes „Bewegungsapparat“ aus der Pareto-Reihe Radiologie gewinnen: Beantworten Sie dazu die Fragen zu diesem Artikel bis zum 31. Juli 2016.

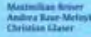

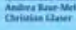

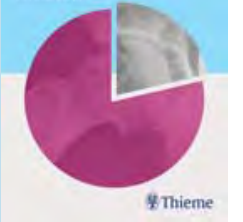




\section{CRTE/CPD-Fragen Konventionelle Röntgendiagnostik der Patella: Einstelltechnik, Tipps und Tricks}

\section{1}

A. Die Patella ist das größte Sesambein im menschlichen Körper.

B. Die Patella besteht aus hyalinem Knorpel.

C. Die Patella gleitet im Gleitlager.

D. Die Patella hat eine Spitze und einen Pol.

E. Die Patella ist in die Sehne des M. quadriceps eingebettet.

\section{Die Röhrenkippung bei der Patella tangential ist}

A. immer $30^{\circ}$.

B. immer $60^{\circ}$.

C. immer $90^{\circ}$.

D. immer $45^{\circ}$.

E. immer entlang des Retropallarspalts.

\section{3}

A. $30^{\circ}, 60^{\circ}, 90^{\circ}$ durchgeführt.

B. $20^{\circ}, 40^{\circ}, 90^{\circ}$ durchgeführt.

C. $30^{\circ}, 45^{\circ}, 90^{\circ}$ durchgeführt.

D. $10^{\circ}, 20^{\circ}, 30^{\circ}$ durchgeführt.

E. $40^{\circ}, 50^{\circ}, 60^{\circ}$ durchgeführt.

\section{Welche Aussage ist nicht richtig?}

A. Die Lagerung des Patienten muss achsgerecht erfolgen.

B. Bei der Aufnahme nach Merchant sollten die Beine mit einem Band zusammengebunden werden.

C. Die Aufnahme nach Merchant eignet sich zum Ausschluss einer Patellalluxation.

D. Bei einer Patellaquerfraktur muss eine Patella tangential angefertigt werden.

E. Der Zentralstrahl wird nach der Patellarückfläche gekippt.

5 Welche Aussage trifft zu? Der Strahlenschutz bei der Patella tangential

A. muss zwischen die Beine gedrückt werden.

B. ist unerheblich.

C. beschränkt sich auf die Schilddrüse.

D. ist nur bei Schwangerschaft erforderlich.

E. sollte immer weggelassen werden, da wichtige Strukturen verdeckt werden können.

\author{
6 Die Röntgenaufnahme nach Merchant wird immer mit \\ einer Kniebeugung von \\ A. $30^{\circ}$ durchgeführt. \\ B. $60^{\circ}$ durchgeführt. \\ C. $90^{\circ}$ durchgeführt. \\ D. $45^{\circ}$ durchgeführt. \\ E. $0^{\circ}$ durchgeführt.
}

7 Welche Aussage trifft zu?

A. Der Vastus medialis zieht die Patella nach außen oben.

B. Die Patella ist in die Sehne des M. quadriceps eingebettet.

C. Die Patellasehne setzt am Patellapol an.

D. Die Patella ist das kleinste Sesambein im Körper.

E. Der Vastus lateralis zieht die Patella nach innen unten.

8 Die verschiedenen Ausformungen der Patella werden eingeteilt nach

A. Merchant.

B. Wilsberg.

C. Wiberg.

D. Willberg.

E. Wisberg.

9 Bei der Patella Defilée muss der Retropatellarspalt

A. nicht zur Darstellung kommen.

B. komplett einsehbar sein.

C. nur teilweise einsehbar sein.

D. nicht beurteilbar sein.

E. nur bei Fraktur zur Darstellung kommen.

10 Welche Aussage trifft zu?

A. Die Einstellung der Patella tangential in Bauchlage ist aus Strahlenschutzgründen der Einstellung in Rückenlage vorzuziehen.

B. Die Einstellung der Patella tangential nach Wilsberg ist zur Diagnostik einer Patellaluxation geeignet.

C. Die Einstellung nach Merchant ist zur Diagnostik einer Patellafraktur unabdingbar.

D. Eine achsgerechte Lagerung des Patienten ist zu vernachlässigen.

E. Die Belichtung der Patella tangential erfolgt immer mit $90 \mathrm{kV}$ und $5 \mathrm{mAs}$. 